\title{
Railway Capacity Calculation in Emergency Using Modified Fuzzy Random Optimization Methodology
}

\author{
Li Wang, Min An, Limin Jia and Yong Qin
}

1 Abstract Accurate estimated capacity of the railway section can provide reliable information to railway operators and engineers in decision-making, particularly, in an emergency situation. However, in an emergency, the optimization of capacity of a railway section is usually involved to study, for example, the characteristics of dynamic, fuzziness, randomness, and non-aftereffect properties. This paper presents a proposed capacity calculation method based on the modified fuzzy Markov chain (MFMC). In this method, the capacity of a railway section in an emergency can be expressed by a fuzzy random variable, which remains the randomness of capacity changing according to the impact of emergencies and the fuzziness of the driving behavior and other factors. A case study of a high-speed line from Beijing to Shanghai is used to show the process of the proposed methods for optimization of section capacity calculation in an emergency.

Keywords Railway section capacity $\cdot$ High-speed railway $\cdot$ Fuzzy Markov chain Emergency

L. Wang

School of Traffic and Transportation, Beijing Jiaotong University, Beijing, China

L. Jia · Y. Qin

State Key Laboratory of Rail Traffic Control and Safety, Beijing Jiaotong University, Beijing 100044, China

M. An (凶)

School of Science, Engineering and Environment, University of Salford, Salford M5 4WT, UK e-mail: M.An@salford.ac.uk

L. Wang $\cdot$ L. Jia $\cdot$ Y. Qin

Beijing Engineering Research Center of Urban Traffic Information Intelligent Sensing and Service Technologies, Beijing 100044, China 


\section{Introduction}

Railway emergency can be defined as the railway system will be affected by different types and levels of failures or incidents [1, 2], such as track system failure, signal system failure, and even an accident. Capacity calculation of a railway section and rerouting path generation in an emergency are usually the basis for the planned subsequent adjustment scheme which includes service planning [3], rolling-stock planning, and timetabling [4-6]. Railway section capacity refers to the maximum number of the trains running on the railway section in a contain time (day, or night or even if several hours) under the conditions of certain train types and the traffic organization. Its influence factors mainly include the number of sections, the condition of railway signal systems, speed of trains, length of block sections, and train sequences [7]. The current methods used to assess capacity of the railway section include graphic methods [8], analytical methods [9], and optimization methods [10]. Since the section capacity calculation involves many factors, the mathematical model or computer simulation only considered the key factors that cannot be completed in conformity with the reality situation. Especially, in emergency conditions, the railway system will be affected by different types and levels of failures or incidents, so that the transport will be interrupted or a train will be limited to a low speed in a certain section. Therefore, it is essential to develop new methods that can take these factors into consideration to provide more accurate capacity estimation in emergency conditions, so that optimization and rerouting path can be undertaken to find the best solution.

\section{Factor Analysis of Railway Section Headway}

The headway of railway section is very important in safe railway operation, which controls the capacity of railway sections. Usually, there are two headway block modes, i.e., the quasi-moving block mode and the fixed block mode, which are described in the below sections.

\subsection{Train Headway Under Fixed Block Mode}

Fixed block mode defines that a section can be divided into a number of sub-sections, and each sub-section has a fixed distance space. As can be seen $L_{s}^{\prime}, L_{s}^{\prime \prime}, L_{s}^{\prime \prime \prime}$ as shown in Fig. 1 are three-fixed blocks, which are recognized by signals. The train operation depends on the signal control modes, such as three- and four-patterns that are mostly used in control operation in railway systems.

In the three-pattern fixed block section, the headway interval of the train usually separates in three-pattern block areas (see Fig. 1), in which Trains $A$ and $B$ have a 
Fig. 1 Three-pattern fixed block mode

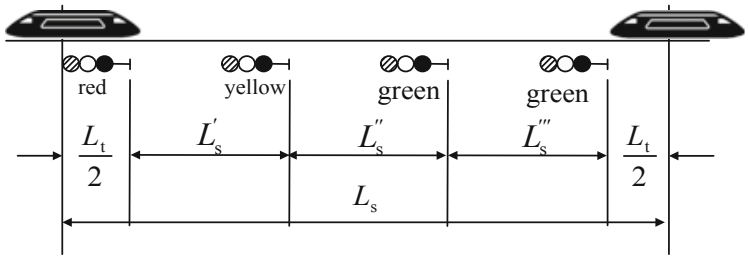

same length; $L_{t}$ is length of train; $L_{s}^{\prime}, L_{s}^{\prime \prime}, L_{s}^{\prime \prime \prime}$ are three-block section intervals length, respectively, (m); the calculation of the train headway expression is shown in Eq. 1.

$$
I=\left(L_{t}+L_{s}^{\prime}+L_{s}^{\prime \prime}+L_{s}^{\prime \prime \prime}\right) / v \times 3.6
$$

In the four-pattern fixed block section, the headway interval of the train usually separates in four-pattern block areas, as shown in Fig. 2. Assume $L_{s}^{\prime}=L_{s}^{\prime \prime}=L_{s}^{\prime \prime \prime}=$ $L_{s}$, the train headway can be calculated by Eq. 2 .

$$
I=\left(4 L_{\text {sunsection }}+L_{\text {train }}\right) / v \times 3.6
$$

In fixed block mode, the train headway mainly depends on the length of the block section and the train operation speed. Normally, the three-pattern block section requires whose length of one block section to satisfy one train barking distance. And the four-pattern block section requires two- or three-block sections. The length of the block sections is determined by the train braking distance, signaling indication system, and the safety redundancy. However, the main factors that affect the braking distance are the traction machines, traction weight, route speed restriction, route slope, etc. These factors increase the uncertainty of the train headway under the fixed block mode. The length of the block section can choose from the range 1600-2600 m under the three-pattern mode and the 700-900 m under the four-pattern mode. In this case, the parameter $L_{s}$ is a fuzzy value and denoted as $\widetilde{L}_{s}$ (in this study, superscript " " expresses that a parameter is a fuzzy parameter). The average train speed $v$ is affected by, for example, rail conditions, the driver behavior, the states of equipment, and the external environmental influence. During an emergency period, the average train speed $v$ is varied fuzzily and randomly, which can be denoted as $\hat{v}$ (in this study,

\begin{tabular}{l|c|c|c|c|c} 
Train 1 & $-H^{G}$ & $G$ & $Y$ & $R$ \\
\hline Block Area & $\begin{array}{c}\text { Attention } \\
\text { Area }\end{array}$ & $\begin{array}{c}\text { Braking } \\
\text { Area 1 }\end{array}$ & $\begin{array}{c}\text { Braking Area } \\
2\end{array}$ & $\begin{array}{c}\text { Braking } \\
\text { Area 3 }\end{array}$ & $\begin{array}{c}\text { Occupation } \\
\text { Area }\end{array}$ \\
\hline Information & Attention & Notice & Notice & Stop &
\end{tabular}

Fig. 2 Four-pattern fixed block mode 
the superscript "“" expresses a parameter is a fuzzy random parameter). Therefore, under the three-pattern mode and four-pattern mode, the calculation function of the train headway with uncertainty can be transformed into Eqs. 3 and 4, respectively.

$$
\begin{gathered}
\widetilde{I}=\left(L_{t}+3 \widetilde{L}_{s}\right) / \hat{v} \times 3.6 \\
\widetilde{I}=\left(L_{t}+4 \widetilde{L}_{s}\right) / \hat{v} \times 3.6 .
\end{gathered}
$$

\subsection{Train Headway Under Fixed Block Mode}

Quasi-moving block mode defines the distance between two trains which is controlled by signals using digital technologies such as GPS and mobile communications. In other words, the distance between two trains is varied depending on train speeds, and rail conditions, the time use of signal system reaction, etc. Currently, high-speed railways adopt the quasi-moving block mode to control the distance between Trains $A$ and $B$ (see Fig. 3). In this case, the time of the train headway can be calculated as Eq. 5.

$$
I=\left(\left(L_{a}+L_{d}+L_{b}+L_{p}+L_{c}+L_{t}\right) / v\right) \times 3.6
$$

where $I$ is the total time of headway that is needed for Train $A$ stopping in order to prevent clash with Train $B, v$ is the average speed $\left(\mathrm{km} \mathrm{h}^{-1}\right)$ of Train $A, L_{a}$ is the distance related to response time, for example, when Train $A$ receives a braking signal, the time is needed for a train driver to take an action, $L_{d}$ is the distance related to the time needed to launch the brake system, $L_{b}$ is the distance from break launch to Train $A$ stopping, $L_{p}$ is the safety distance depending on rail conditions and train speeds, $L_{c}$ is the distance of normal operation, and $L_{s}$ is the total of the distance as shown in Fig. 3.

Equation 5 can be transferred to the speed-time expression as Eq. 6.

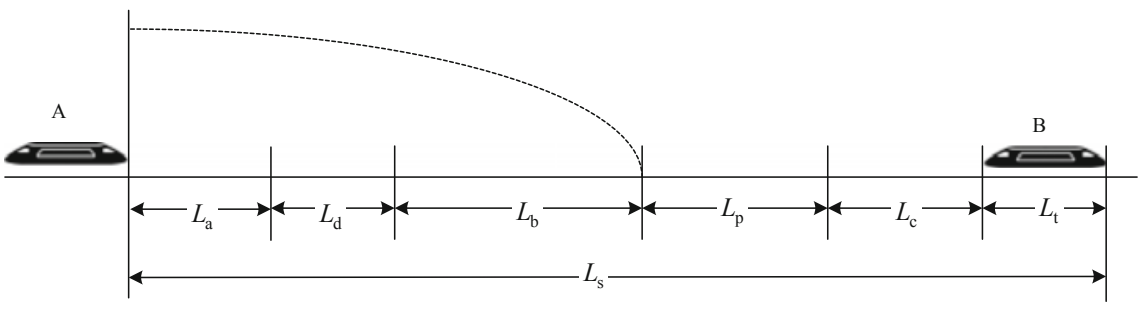

Fig. 3 Quasi-moving block mode 


$$
I=t_{a}+t_{d}+(v / 3.6) / 2 a+\left(L_{p}+L_{c}+L_{t}\right) / v / 3.6
$$

As can be seen from Eq. 6, to determine the parameters depends on, for example, rail conditions, train speeds, types of train control systems, types of the braking systems, and also the driver behaviors, etc. The $t_{a}$ is affected by many factors such as the time of the train control system to response, the time of information transmission, and the reaction time of the driver. Landex [12] studied the $t_{a}$ and believes that $t_{a}$ is around $9.5 \mathrm{~s}$. The $t_{d}$ is the response time of the train brake system. Again, $t_{d}$ is also a fuzzy value, which is generally considered as around $2.5 \mathrm{~s}$ [12]. The $a$ is the braking deceleration speed and is determined based on the braking performance of the train and route conditions. Xianming [13] studied the corresponding deceleration speeds when a train is running on a downhill slope with $20 \%$, plain section and approaching to the station, and $a$ can choose $0.565 \mathrm{~m} \mathrm{~s}^{-2}, 0.75 \mathrm{~m} \mathrm{~s}^{-2}$, and $0.5 \mathrm{~m} \mathrm{~s}^{-2}$, respectively. Because of the differences of the train braking system and the route conditions, the value of $a$ is fuzziness with the range from 0.5 to $0.75 \mathrm{~m} \mathrm{~s}^{-2}$. If the safety margin was considered, the value of $L_{p}$ can choose with the range of 80-150 m; The value of $L_{c}$ changes with the differences of the control mode, the longer the value of $L_{c}$, the drivers' behavior shows more calm down. However, it will increase the train headway at the meantime. Therefore, it will keep the comfortable driving and high usage of the ability by chosen 1-2 block length. Equation 6 can be expressed as Eq. 7

$$
\tilde{I}=\tilde{t}_{a}+\tilde{t}_{d}+\hat{v}^{2} / 2 \tilde{a}+\left(\left(\widetilde{L}_{p}+\tilde{L}_{c}+L_{t}\right) / \hat{v}\right) \times 3.6 .
$$

\section{Factor Analysis of Railway Section Headway}

Based on the analysis of uncertainty of fuzzy and stochastic, the railway section capacity status changing process in emergency is described as a fuzzy Markov chain. Then, the calculation of section capacity is changed to the fuzzy status transfer processing. Finally, the parameters sensitivity analysis is given in the section.

\subsection{Uncertainty Analysis of the Railway Section Capacity}

The severity of the incident influences and the railway department repair ability are changing randomly, which cause a random variation in train operation speed correspondingly. Therefore, the section capacity changes randomly in a period of time. Moreover, in a certain time of future, the railway network capacity is associated with the current equipment instead of the former condition. As the conclusion, the transformation of the section capacity has the non-aftereffect property for different rail lines and emergency types. The changing process of the section capacity status can be described as Markov chain (MC) [14]. Due to the fuzziness property of the 


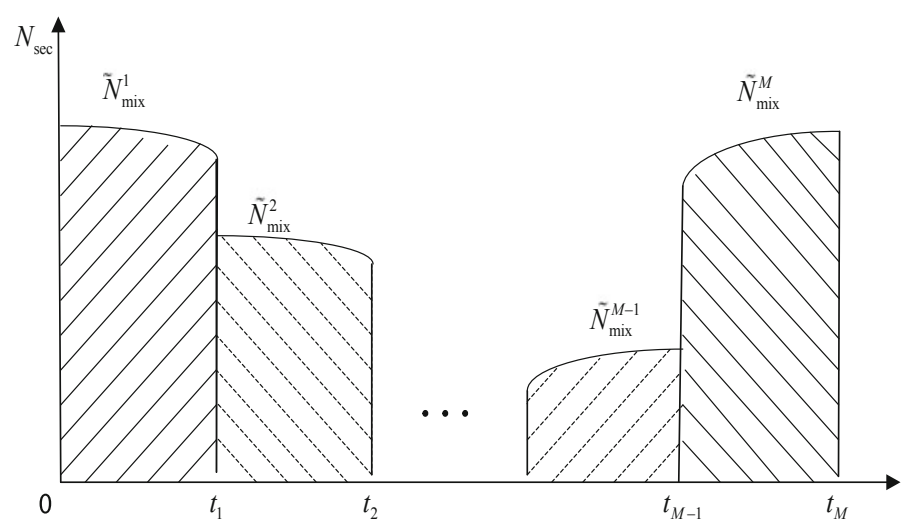

Fig. 4 Section capacity changing process in an emergency

section capacity itself, the capacity status changing process is considered as the fuzzy Markov chain (FMC) in this paper. The definition of FMC is given in Sect. 3.2.

As shown in Fig. 4, the time axis is divided into $M$ periods. Because of the different effect level of the special events in each period, the different emergency grade, the different degree of the maintenance, and the train speed limitation are different. So, the capacity of each time period is also different. Then, the whole section capacity can be computed by Eq. 8 . Where, $\widetilde{N}_{\text {mix }}^{i}$ is fuzzy value of the section capacity in the $i$ th period, by setting $\Delta t^{(i)}=t_{i}-t_{i-1}, i=1,2, \ldots, M$.

$$
N_{\text {sec }}=\sum_{i=1}^{M} \widetilde{N}_{\text {mix }}^{i}
$$

The dividing of the time periods depends on the influence degree of the incidents and the railway line recovery levels. Therefore, the dividing has property of fuzziness. In reality, it can be determined by the development of the incident and the speed limited strategy.

\subsection{Fuzzy Markov Chain}

The fuzzy random variable (FRV) is a measurable function, which is the set from the probability space mapping to the fuzzy space. For example, for a given probability space $\{\Theta, \Lambda, \operatorname{Pr}\}, \tilde{u}_{1}, \tilde{u}_{2}, \ldots, \tilde{u}_{n}$ are the fuzzy variable, if $\xi\left(\omega_{i}\right)=\tilde{u}_{i}, i=$ $1,2, \ldots, n$, then, $\xi\left(\omega_{i}\right)$ is the fuzzy random variable [15]. Figure 5 shows the relationship among an FRV, a random variable, and a fuzzy variable.

Definition 1 Fuzzy Markov Chain (FMC) For a given probability space $(\Omega, \Lambda$, $P$ ), $\Theta$ is nonempty set, $\Lambda$ is the $\sigma$ algebra of $\Gamma$, and $P$ is the probability. The number 


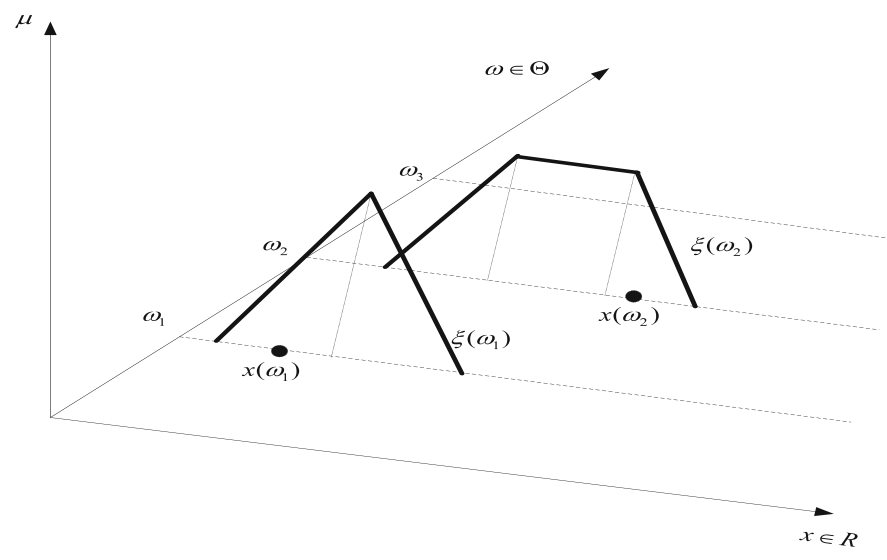

Fig. 5 FRV graph

of the fuzzy random variable $\{\tilde{X}(t), t=0,1,2, \ldots\}$ is limited or countable. For the whole possible fuzzy conditions of all $\widetilde{X}(t)$, there is one group of fuzzy set $\widetilde{A}=\left\{\widetilde{A}_{0}, \widetilde{A}_{1}, \widetilde{A}_{2}, \ldots\right\}$ corresponding to $\widetilde{X}(t)$, make $S$ to the possibility of one fuzzy event, if the possibility of $\widetilde{X}(n+1)=\widetilde{A}_{n+1}$ (variable $\widetilde{X}(n+1)$ at the moment $n+1$ stay in the $\widetilde{A}_{n+1}$ status) can only relate to $\widetilde{X}(n)$, but not the status before the $n$, expressed as $S\left(\widetilde{X}(n+1)=\widetilde{A}_{n+1} \mid \widetilde{X}(n), \widetilde{X}(n-1), \ldots, \widetilde{X}(0)\right)=S\left(\widetilde{X}(n+1)=\widetilde{A}_{n+1} \mid \widetilde{X}(n)\right)$, thus, the sequence $\widetilde{X}(t)$ is named as fuzzy Markov chain (FMC).

Signed $S$ as one-step transfer possibility matrix, which is denoted by Eq. 9 .

$$
S=\left[\begin{array}{ccccc}
s_{0,0} & s_{0,1} & \cdots & s_{0, j} & \cdots \\
\cdots & \cdots & \cdots & \cdots & \cdots \\
s_{i, 0} & s_{i, 1} & \cdots & s_{i, j} & \cdots \\
\cdots & \cdots & \cdots & \cdots & \cdots
\end{array}\right] .
$$

\subsection{Calculation of Section Capacity Fuzzy Value}

Assuming in the continuous emergency time $T$, the speed type of the train is $Q_{\text {spe }}$, denoted as $v^{(1)}, v^{(1)}, \ldots, v^{\left(Q_{\text {spe }}\right)}$. Divide the continuous time $T$ into $Q_{c}$ time periods, the correspond section capacity has $Q_{c}$ kind of situations, denoted as $\tilde{c}_{1}, \tilde{c}_{2}, \ldots$, $\tilde{c}_{i}, i=1,2, \ldots, Q_{c}$, consisting all the possible fuzzy sets $\widetilde{C}$ of the capacity changing Markov chain procession.

Firstly, the section capacity can be calculated in one time period by Eq. 10 .

$$
c=\left(60 \times 60-t_{\text {mai }}\right) / I
$$


Here, $c$ is the section capacity in each time period. $t_{\text {mai }}$ is the comprehensive maintenance time. If different trains' speeds exist, the capacity can be calculated by the deduction coefficient method.

The status transfer matrix of train speed can be denoted by Eq. 11 .

$$
P=\left[\begin{array}{cccc}
P_{1,1} & P_{1,2} & \cdots & P_{1, Q_{\text {speed }}} \\
P_{2,1} & P_{2,2} & \cdots & P_{2, Q_{\text {speed }}} \\
\vdots & \vdots & \ddots & \vdots \\
P_{Q_{\text {speed }}, 1} & P_{Q_{\text {speed }}, 2} & \cdots & P_{Q_{\text {speed }}, Q_{\text {speed }}}
\end{array}\right]
$$

Here, $P_{i, j}$ is the probability of the train speed from $v^{(i)}$ to $v^{(j)}$. Then, set the status transfer matrix of the section capacity under the possible measurement can be calculated by Eq. 12 .

$$
S=\left[\begin{array}{cccc}
s_{1,1} & s_{1,2} & \cdots & s_{1, Q_{c}} \\
s_{2,1} & s_{2,2} & \cdots & s_{2, Q_{c}} \\
\vdots & \vdots & \ddots & \vdots \\
s_{Q_{c}, 1} & s_{Q_{c}, 2} & \cdots & s_{Q_{c}, Q_{c}}
\end{array}\right]
$$

Here, $s_{i, j}$ is the condition probability of the section capacity from $\tilde{c}_{i}$ to $\tilde{c}_{j}$ under the possible measurement [16], denoted as Eq. 13.

$$
s\left(\tilde{c}_{j} / \tilde{c}_{i}\right)=s\left(\tilde{c}_{j}, \tilde{c}_{i}\right) / s\left(\tilde{c}_{i}\right)
$$

For the convenient of calculation, define matrix $Q_{1}$ and $Q_{2}$ by Eqs. 14 and 15 .

$$
\begin{gathered}
Q_{1}=\left[\begin{array}{cccc}
\mu_{\tilde{c}_{1}}(0) & \mu_{\tilde{c}_{2}}(0) & \cdots & \mu_{\tilde{c}_{N}}(0) \\
\mu_{\tilde{c}_{1}}(1) & \mu_{\tilde{c}_{2}}(1) & \cdots & \mu_{\tilde{c}_{N}}(1) \\
\vdots & \vdots & \ddots & \vdots \\
\mu_{\tilde{c}_{1}}(N) & \mu_{\tilde{c}_{2}}(N) & \cdots & \mu_{\tilde{c}_{N}}(N)
\end{array}\right] \\
Q_{2}=\left[\begin{array}{cccc}
p_{0} \mu_{\tilde{c}_{1}}(0) / P\left(\tilde{c}_{1}\right) & p_{1} \mu_{\tilde{c}_{1}}(1) / P\left(\tilde{c}_{1}\right) & \cdots & p_{N} \mu_{\tilde{c}_{1}}(N) / P\left(\tilde{c}_{1}\right) \\
p_{0} \mu_{\tilde{c}_{2}}(0) / P\left(\tilde{c}_{2}\right) & p_{1} \mu_{\tilde{c}_{2}}(1) / P\left(\tilde{c}_{2}\right) & \cdots & p_{N} \mu_{\tilde{c}_{2}}(N) / P\left(\tilde{c}_{2}\right) \\
\vdots & \vdots & \ddots & \vdots \\
p_{0} \mu_{\tilde{c}_{N}}(0) / P\left(\tilde{c}_{N}\right) & p_{1} \mu_{\tilde{c}_{N}}(1) / P\left(\tilde{c}_{N}\right) & \cdots & p_{N} \mu_{\tilde{c}_{N}}(N) / P\left(\tilde{c}_{N}\right)
\end{array}\right]
\end{gathered}
$$

Therefore, we can get Eq. 16.

$$
s\left(\tilde{c}_{j} / \tilde{c}_{i}\right)=Q_{2} P_{m l} Q_{1}
$$

For all periods, section capacity can be generalized as Eq. 17. 


$$
\left(\widehat{N}_{\text {mix }}^{(l)}\right)^{\mathrm{T}}=\left(\tilde{c}_{1}, s_{h, 1}, \tilde{c}_{2}, s_{h, 2} \cdots \tilde{c}_{Q_{c}}, s_{h, Q_{c}}\right), \quad l=h+1
$$

Therefore, the section capacity of the divide-period in limited speed condition $\widehat{N}_{\text {mix }}^{(i)}$ is the fuzzy random variable.

The section capacity in the whole time $T$ is the sum of all periods of capacity, denoted by Eq. 18.

$$
N_{\mathrm{sec}}=\sum_{i=1}^{Q_{c}} \tilde{N}_{\mathrm{mix}}^{i}=\left\{\begin{array}{l}
\sum_{i=1}^{Q_{c}} \tilde{c}_{1}, \sum_{i=1}^{Q_{c}} s_{i, 1} \\
\vdots \\
\sum_{i=1}^{Q_{c}-1} \tilde{c}_{Q_{c}}+\tilde{c}_{Q_{c}-1}, \sum_{i=1}^{Q_{c}-1} s_{i, Q_{c}} \cdot s_{Q_{c}, Q_{c}-1} \\
\sum_{i=1}^{Q_{c}} \tilde{c}_{Q_{c}}, \sum_{i=1}^{Q_{c}} s_{i, Q_{c}}
\end{array}\right.
$$

It is noticeable to see that in possible measurement the section carrying capacity is still a fuzzy random value in the continuous duration $T$ under the emergency condition.

\section{Case Study}

\subsection{Emergency Scenario}

In this paper, the real-world case study is based on Shanghai to Nanjing section. Figure 6 describes the network of this section and its nearby railways. The Shanghai to Nanjing intercity line and the Beijing to Shanghai existing line are both parallel to Beijing to Shanghai high-speed rail line. In Fig. 6, these three routes are distinguished by the imaginary line, the dash-dot line, and the solid line, respectively. The distance between any two stations is given in Fig. 6. The details of design and train schedules of Shanghai to Nanjing section can be found in [17].

Suppose that the broken-down situation occurs in Suzhou to Kunshan section in Beijing to Shanghai high-speed rail line at 8 a.m., i.e., no train service can provide. Recovery is expected for $3 \mathrm{~h}$. In Zhenjiang West to Wuxi East section, due to the heavy wind situation, speed restriction is implemented. The wind speed is expected to keep at about $20 \mathrm{~m} \mathrm{~s}^{-1}$ for $2 \mathrm{~h}$. Then, the wind speed would increase at about $24 \mathrm{~m} \mathrm{~s}^{-1}$ for $5 \mathrm{~h}$. At last, the wind speed would decrease and back to normal operation environment in $2 \mathrm{~h}$. Besides, other sections and stations are not affected by the emergency. 


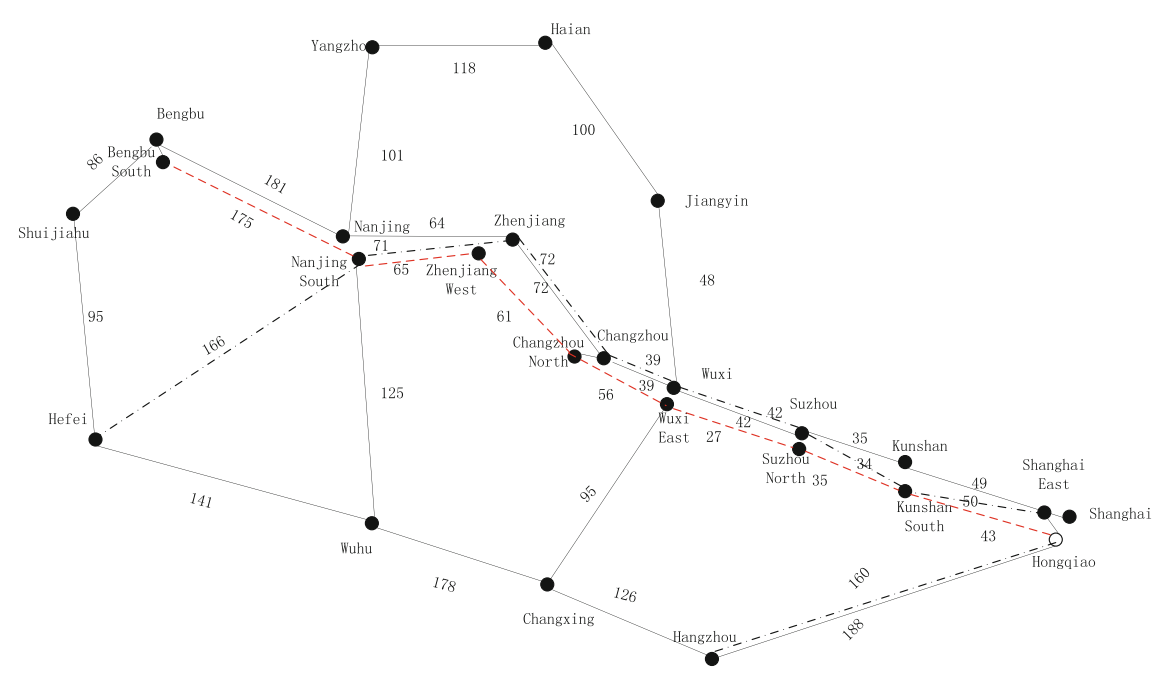

Fig. 6 Part of China railway network

\subsection{Section Capacity Calculation}

In the broken-down section, the capacity can be set to zero directly. Therefore, the capacity calculation is focused on the speed restriction conditions. According to emergency development, the section capacity changing process can be divided into three stages based on the wind speed variation. Based on the railway traffic safety specification, in the above scenario, there are three kinds of train operation speed, $160 \mathrm{~km} \mathrm{~h}^{-1}, 70 \mathrm{~km} \mathrm{~h}^{-1}$, and normal speed. Firstly, calculate the section capacity in different limited speeds. In this period, it only involved of the medium and high-speed trains, therefore, when the train recovered to normal operation, the average speed of the train can be expressed as the fuzziness value $\tilde{v}_{0}=\left(330 \mathrm{~km} \mathrm{~h}^{-1}\right.$, $340 \mathrm{~km} \mathrm{~h}^{-1}, 350 \mathrm{~km} \mathrm{~h}^{-1}$ ). In addition, the average speed of the train changes due to the environment, the operation equipment conditions, and the drivers' driving skills [18]. Normally, the average speed changes in one range and smaller than the limited speed. Therefore, when the limited speed is $160 \mathrm{~km} \mathrm{~h}^{-1}$, the average speed of a train can be set as $\tilde{v}_{1}=\left(130 \mathrm{~km} \mathrm{~h}^{-1}, 140 \mathrm{~km} \mathrm{~h}^{-1}, 155 \mathrm{~km} \mathrm{~h}^{-1}\right)$. When the limited speed is $70 \mathrm{~km} \mathrm{~h}^{-1}$, the average speed can be set as $\tilde{v}_{2}=\left(50 \mathrm{~km} \mathrm{~h}^{-1}, 60 \mathrm{~km} \mathrm{~h}^{-1}, 65 \mathrm{~km} \mathrm{~h}^{-1}\right)$. Calculate the section capacity value in different speed restriction conditions. We can set $t_{a}$ as $9.5 \mathrm{~s}, t_{d}$ as $2.5 \mathrm{~s}, a$ as $0.7 \mathrm{~m} \mathrm{~s}^{-2}, L_{p}$ as $150 \mathrm{~m}$ according to Sect. 2. The value of $L_{c}$ relates to the train speed, when the train is with high speed, it can take the length of two-block section, which is about $4000 \mathrm{~m}$; when the train is running in lower speed, $L_{c}$ can take the length of one block section, which is about $2000 \mathrm{~m}$. $L_{t}$ can be set to the conservative value $400 \mathrm{~m}$. Then, put all of the parameters into Eq. 2, we getting the following results. 
When the train operated normally, the train headway $\widetilde{I}_{0}$ is $(124,127,131)$. Section capacity $\tilde{c}_{0}$ can be calculated by using Eq. (10) is $(27,28,29)$. When the limited speed is $160 \mathrm{~km} \mathrm{~h}^{-1}$, the train headway $\widetilde{I}_{1}$ is $(143,156,168), \tilde{c}_{1}$ is $(21,23,25)$. When the limited speed is $70 \mathrm{~km} \mathrm{~h}^{-1}, \widetilde{I}_{2}$ is $(163,187,208), \tilde{c}_{2}$ is $(17,19,22)$.

\section{Calculate the capacity transfer conditional probability in emergency condition.}

Assume the first stage of the train's limited speed is $160 \mathrm{~km} \mathrm{~h}^{-1}$. We can get the first stage section capacity $\widehat{N}_{\text {mix }}^{(1)}$ by Eq. 19 .

$$
\widehat{N}_{\text {mix }}^{(1)}=2 \tilde{c}_{1}=(42,46,50)
$$

In order to make the calculation convenience, assume $v_{0}=330, v_{1}=340, v_{2}=$ $350, v_{3}=130, v_{4}=140, v_{5}=155, v_{6}=50, v_{7}=60, v_{8}=65$. The probabilities of different speed $p_{v_{i}}$ are set to the same, $p_{v_{i}}=1 / 9, i=1,2, \ldots, 8$. The capacity transfer condition probability of the second stage and the third stage can be obtained based on Eq. 12. The transfer matrixes are denoted as $S^{1}$ and $S^{2}$.

$$
\begin{array}{r}
S^{1}=\left[\begin{array}{lll}
0.2000 & 0.7833 & 0.0167 \\
0.1248 & 0.7891 & 0.0861 \\
0.0079 & 0.1566 & 0.8355
\end{array}\right] \\
S^{2}=\left[\begin{array}{lll}
0.8667 & 0.1317 & 0.0016 \\
0.6097 & 0.3758 & 0.0145 \\
0.0207 & 0.9017 & 0.0776
\end{array}\right]
\end{array}
$$

Sum divided-period capacities. According to section capacity calculation method based on FMC in Sect. 3, the carrying capacity of the second stage and third stage can be expression as the fuzziness random variable, particular form shown as:

$$
\begin{aligned}
& \widehat{N}_{\text {mix }}^{(2)}=\left\{\begin{array}{ll}
5 \tilde{c}_{0}, s_{1,0}^{1} \\
5 \tilde{c}_{1}, s_{1,1}^{1} \\
5 \tilde{c}_{2}, s_{1,2}^{1}
\end{array}= \begin{cases}(135,140,145), & 0.1248 \\
(105,115,125), & 0.7890 \\
(85,95,110), & 0.0861\end{cases} \right. \\
& \widehat{N}_{\text {mix }}^{(3)}=\left\{\begin{array}{l}
2 \tilde{c}_{0}, s_{1,0}^{2} \\
2 \tilde{c}_{1}, s_{1,1}^{2} \\
2 \tilde{c}_{2}, s_{1,2}^{2}
\end{array}=\left\{\begin{array}{l}
(54,56,58), 0.6097 \\
(42,46,50), 0.3758 \\
(34,38,44), 0.0145
\end{array}\right.\right.
\end{aligned}
$$

Therefore, the total capacity for nine hours is calculated by Eq. 18 . 


$$
\widehat{N}_{\mathrm{sec}}=\sum_{i=1}^{3} \widehat{N}_{\text {mix }}^{(i)}=\left\{\begin{array}{l}
(231,242,253), 0.0761 \\
(219,232,245), 0.0469 \\
(211,224,239), 0.0018 \\
(201,217,233), 0.4811 \\
(189,207,225), 0.2965 \\
(181,199,219), 0.0114 \\
(181,197,218), 0.0525 \\
(169,187,210), 0.0324 \\
(161,179,204), 0.0013
\end{array}\right.
$$

It can be found from Eq. 36, the expectancy value of the comprehensive capacity is 215.197 . The calculation process of the comprehensive capacity expectation explained that in the current scenario the section capacity is about 215 . The possible maximum and minimum values are 242 and 180, respectively. This provides alternative offers for the policymaker to make the decision depended on the different preferences. If the radical arrangement was chosen, the value can set to 242 . However, if the operator preferred to the conservative strategy, the main target is to meet the basic demand of the transportation, the value can set to 180 . Generally, the compromise strategy is adopted, which means the value sets to 215 .

This paper focuses on the railway section capacity calculation, without the station capacity. In this case, the heavy wind affects train operation speed running on section mainly, but less effect on the station. Therefore, the section capacity decides the line capacity at current scenario.

\section{Conclusion and Future Work}

Section capacity calculation involves multiple factors and complicated relationships. In emergency conditions, the factors of the calculation capacity present the characteristics of dynamic, fuzziness, randomness, no aftereffect, etc. In the reality, the capacity calculation cannot include all of the factors. FMC-based capacity calculation method can be more fault-tolerant of all kinds of sensitive factors and the uncertainties. In addition, this method provides variable choices for the policymakers, so that it can suit to radical or conservative strategies.

The research in the future is mainly concentrated in two aspects: (1) the train speed transfer matrix is the key factor to realize the section capacity calculation, the value of the transfer matrix need to be confirmed in the practical operation environment; (2) there is a big difference of train operation objectives, strategies, and principles between in the emergency and in the normal conditions. Line planning, timetable rescheduling, and rolling-stock rebalancing are yet to consider together in emergency condition. Our ultimate goal is to design and develop a real-time decision support framework in the future, which will decrease the influence of the emergency effectively and recover the train operation quickly. 
Acknowledgments This study is funded by the National Key Research and Development Program of China (2016YFB1200401) and National Natural Science Foundation of China (71701010).

\section{References}

1. Xu XN, Zhuang L, Xing B et al (2018) An ultrasonic guided wave mode excitation method in rails. IEEE Access 6:60414-60428

2. Kou L, Qin Y, Zhao X et al (2019) Integrating synthetic minority oversampling and gradient boosting decision tree for bogie fault diagnosis in rail vehicles. Proc Inst Mech Eng Part F: J Rail Rapid Transit 233(3):312-325

3. Meng XL, Qin Y, Jia LM (2014) Comprehensive evaluation of passenger train service plan based on complex network theory. Measurement 58:221-229

4. Meng XL, Jia LM, Xiang WL (2018) Complex network model for railway timetable stability optimisation. IET Intell Transp Syst 12(10):1369-1377

5. Zhang HR, Jia LM, Wang L et al (2019) Energy consumption optimization of train operation for railway systems: algorithm development and real-world case study. J Clean Prod 214:1024 1037

6. Wang MM, Wang L, Xu XY et al (2019) Genetic algorithm-based particle swarm optimization approach to reschedule high-speed railway timetables: a case study in China. J Adv Transp

7. Abril M, Barber F, Ingolotti L, Salido M, Tormos P, Lova A (2008) An assessment of railway capacity. Transp Res Part E: Logist Transp Rev 44:774-806

8. Riejos FAO, Barrena E, Ortiz JDC, Laporte G (2016) Analyzing the theoretical capacity of railway networks with a radial-backbone topology. Transp Res Part A: Policy Pract 84:83-92

9. Zhang X, Nie L (2016) Integrating capacity analysis with high-speed railway timetabling: a minimum cycle time calculation model with flexible overtaking constraints and intelligent enumeration. Transp Res Part C: Emerg Technol 68:509-531

10. Petering MEH, Heydar M, Bergmann DR (2016) Mixed-integer programming for railway capacity analysis and cyclic, combined train timetabling and platforming. Transp Sci 50:892909

11. Zhang YG, Zeng QF, Lei DY, Wang XY (2016) Simulating the effects of noncrossing block sections setting rules on capacity loss of double-track railway line due to the operation of out-of-gauge trains. Discret Dyn Nat Soc

12. Landex A, Kaas AH, Schittenhelm B, Schneider-Tilli J (2006) Evaluation of railway capacity. In: Annual transport conference at Aalborg University, Aalborg, pp 1-22

13. Xianming S (2005) Research on the headway time of passenger train in China. China Railway Sci 7:32-35 (in Chinese)

14. Wang L, Qin Y, Xu J et al (2013) Section carrying capacity calculation in emergency for high-speed railway. J Beijing Inst Univ S1:18-21

15. Liu B (2007) Uncertainty theory. Springer, Berlin

16. Pardo MJ, de la Fuente D (2010) Fuzzy Markovian decision processes: application to queueing systems. Comput Math Appl 60:2526-2535

17. Wang L, Jia LM, Qin Y et al (2011) A two-layer optimization model for high-speed railway line planning. J Zhejiang Univ: Sci A 12:902-912

18. Wang L, Qin Y, Xu J, Jia L (2012) A fuzzy optimization model for high-speed railway timetable rescheduling. Discret Dyn Nat Soc 